\title{
Slug enhances invasion ability of pancreatic cancer cells through upregulation of matrix metalloproteinase-9 and actin cytoskeleton remodeling
}

Kejun Zhang, Dong Chen, Xuelong Jiao, Shaoyan Zhang, Xiangping Liu, Jingyu Cao, Liqun Wu and Dongsheng Wang

Laboratory Investigation (2012) 92, 1801; doi:10.1038/labinvest.2012.138; published online 12 November 2012

Retraction to: Laboratory Investigation (2011) 91, 426-438; doi:10.1038/labinvest.2010.201; published online 31 January 2011

The authors of this article have requested its retraction from Laboratory Investigation because of their inability to reproduce key experiments:

Zhang K, Chen D, Jiao X, Zhang S, Liu X, Cao J, Wu L, Wang D. Slug enhances invasion ability of pancreatic cancer cells through upregulation of matrix metalloproteinase- 9 and actin cytoskeleton remodeling. Lab Invest 2011;91:426-438.

This paper reports that Slug transfection does not affect E-cadherin expression. However, upon repeating the experiment, we found Slug transfection significantly reduces the E-cadherin expression. Additionally, MMP-2 was upregulated in new experiments.

The paper also reveals that intracellular F-actin and MMP9 levels are increased and relocated to the tip of the extending pseudopodia from the perinuclear pool in Slug-transfected PANC-1 cells. However, upon repeating the experiment, it appeared that only F-actin, not MMP-9, was relocated to the tip of the extending pseudopodia. Therefore, the cellular localization of the MMP-2 cells needs further investigation.

Furthermore, there were several minor errors in the paper:

1. The primer sequence for E-cadherin should be $5^{\prime}$-GGAA GTCAGTTCAGACTCCAGCC- $3^{\prime}$ and $5^{\prime}$-AGGCCTTTTGA CTGTAATCACACC-3'; not $5^{\prime}$-TTCAGTTCCGAGGTCTA CAC-30; antisense: 30-GTCTCTGTGGTGATGCCGGT-5, as was reported.

2. The wrong reference was cited for the BB94 treatment. The correct concentration was $0.25 \mu \mathrm{mol} / \mathrm{l}$, not $0.1 \mathrm{umol} / \mathrm{l}$, as was written.

3. Female C57BL/6 mice were at $4-6$ weeks, not $6-8$ weeks of age.

4. All statistical analyses were performed using SPSS 7.0 software, not SPSS 13.

The authors regret the impact that these inconsistencies and errors may have had on other researchers. 